\title{
Clinical nomograms: Is a picture worth a thousand words?
}

\author{
Kaitlin C. McLoughlin, MD, and R. Taylor Ripley, MD
}

\footnotetext{
From the Thoracic and Gastrointestinal Oncology, National Cancer Institute, Center for Cancer Research, National Institutes of Health, Bethesda, Md.

Disclosures: Authors have nothing to disclose with regard to commercial support.

Received for publication Nov 1, 2016; accepted for publication Nov 1, 2016; available ahead of print Dec 8, 2016. Address for reprints: R. Taylor Ripley, MD, Thoracic and Gastrointestinal Oncology Branch, CCR/NCI, Building 10, 4-3952, 10 Center Dr, MSC 1201, Bethesda, MD 20892-1201 (E-mail: Taylor.Ripley@nih.gov). J Thorac Cardiovasc Surg 2017;153:470-1

0022-5223/\$0.00

Published by Elsevier Inc. on behalf of The American Association for Thoracic Surgery http://dx.doi.org/10.1016/j.jtcvs.2016.11.002
}

Nomograms are pictorial devices that synthesize biologic and clinical variables, both categoric and continuous, to determine the probability of a clinical event occurring in a given patient. ${ }^{1}$ These models may provide benefit over TNM staging by avoiding the Halstedian assumption that anatomic disease progression always correlates with worse outcome. Rather, nomograms attempt to provide a finer risk stratification. Nomograms are visually simple but represent complex calculations and require careful development and application. The clinical question, study population, and experimental outcome are all imperative to produce a useful tool.

Asking a clinically relevant and important question is paramount to model development. For example, nomograms have been developed that query the risk of recurrence after sublobar resections, recurrence after tyrosine kinase inhibitor treatment, and risk of invasive pulmonary adenocarcinoma on the basis of imaging. ${ }^{2-7}$ Jin and colleagues $^{8}$ have developed a nomogram to determine the risk of invasive pulmonary adenocarcinoma in patients with solitary, peripheral subsolid pulmonary nodules. This question is particularly relevant with the increase in computed tomography screening programs after publication of the National Lung Screening Trial. ${ }^{9}$ Because these lesions may represent invasive pulmonary adenocarcinoma, noninvasive disease, or a benign lesion, preoperative differentiation can prevent unnecessary interventions and change the operation performed..$^{10}$ Jin and colleagues ${ }^{8}$ constructed a nomogram with computed tomography attenuation, size, spiculation, vascular convergence, pleural tags, and percentage of solid component. They have paired specific and clinically relevant outcome with clinical parameters and shown a correlation with invasive disease.

Nomogram validation is equally important. This validation is ideally external (performed on multiple, separate datasets) to demonstrate reliability for general implementation. However, many nomograms are internally validated because of insufficient patient numbers requiring

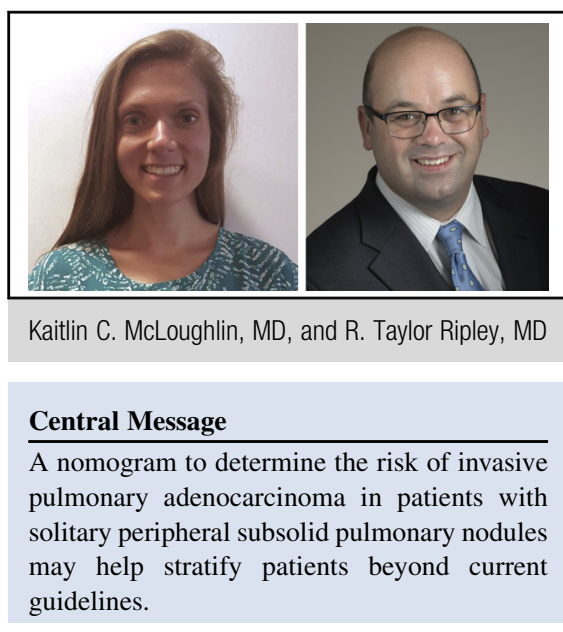

See Article page 462.

multiple iterations of the model applied over variable subsets of the same experimental population. This validation risks overfitting the data that limit the nomogram's generalizability. Jin and colleagues ${ }^{8}$ identified 293 patients and used an internal validation method. Despite the relatively small sample and internal validation, the variables used in this nomogram were similar to those in a larger, externally validated nomogram. She and colleagues ${ }^{11}$ recently reported a nomogram with 898 cases split into a model development cohort and a validation cohort. This nomogram is not a true validation cohort of the nomogram reported by Jin and colleagues. ${ }^{8}$ The terminology is different, such as "speculation" as opposed to "uneasily differentiated margins" and "irregular shape." In addition, the findings of smoking status and solid component did differ between the models. Despite these relatively minor differences, the findings are similar, which suggests that both nomograms may be generalizable.

Is this nomogram clinically useful? Does it improve clinical decisions beyond current guidelines? ${ }^{12}$ For patients with certain subsolid pulmonary nodules, continued surveillance, biopsy, and resection can be equally accepted options according to guideline parameters. In these situations, use of this nomogram may help with clinical decisions. Jin and colleagues ${ }^{8}$ are to be commended for asking a relevant question that can clarify a common clinical scenario. However, with lack of external validation and slight differences from other nomograms, caution should be applied to avoid overuse of this model. 


\section{References}

1. Balachandran VP, Gonen M, Smith JJ, DeMatteo RP. Nomograms in oncology: more than meets the eye. Lancet Oncol. 2015;16:e173-80.

2. Desseroit MC, Visvikis D, Tixier F, Majdoub M, Perdrisot R, Guillevin R, et al. Development of a nomogram combining clinical staging with (18)F-FDG PET/ CT image features in non-small-cell lung cancer stage I-III. Eur J Nucl Med Mol Imaging. 2016;43:1477-85.

3. Keam B, Kim DW, Park JH, Lee JO, Kim TM, Lee SH, et al. Nomogram predicting clinical outcomes in non-small cell lung cancer patients treated with epidermal growth factor receptor tyrosine kinase inhibitors. Cancer Res Treat. 2014;46:323-30.

4. Kent MS, Mandrekar SJ, Landreneau R, Nichols F, Foster NR, DiPetrillo TA, et al. A nomogram to predict recurrence and survival of high-risk patients undergoing sublobar resection for lung cancer: an Analysis of a Multicenter Prospective Study (ACOSOG Z4032). Ann Thorac Surg. 2016;102:239-46.

5. Liang W, Zhang L, Jiang G, Wang Q, Liu L, Liu D, et al. Development and validation of a nomogram for predicting survival in patients with resected non-smallcell lung cancer. J Clin Oncol. 2015;33:860-8.

6. Song SH, Ahn JH, Lee HY, Lee G, Choi JY, Kang J, et al. Prognostic impact of nomogram based on whole tumour size, tumour disappearance ratio on CT and SUVmax on PET in lung adenocarcinoma. Eur Radiol. 2016;26:1538-46.

7. Liang J, Xu XQ, Xu H, Yuan M, Zhang W, Shi ZF, et al. Using the CT features to differentiate invasive pulmonary adenocarcinoma from pre-invasive lesion appearing as pure or mixed ground-glass nodules. Br J Radiol. 2015;88:20140811.

8. Jin C, Cao J, Cai Y, Wang L, Liu K, Shen W, et al. A nomogram for predicting the risk of invasive pulmonary adenocarcinoma for patients with solitary peripheral subsolid nodules. J Thorac Cardiovasc Surg. 2017;153:462-9.

9. National Lung Screening Trial Research Team, Aberle DR, Adams AM, Berg CD, Black WC, Clapp JD, Fagerstrom RM, et al. Reduced lung-cancer mortality with low-dose computed tomographic screening. N Engl J Med. 2011;365: 395-409.

10. Van Schil PE, Asamura H, Rusch VW, Mitsudomi T, Tsuboi M, Brambilla E et al. Surgical implications of the new IASLC/ATS/ERS adenocarcinoma classification. Eur Respir J. 2012;39:478-86.

11. She Y, Zhao L, Dai C, Ren Y, Zha J, Xie H, et al. Preoperative nomogram for identifying invasive pulmonary adenocarcinoma in patients with pure groundglass nodule: a multi-institutional study. Oncotarget. Aug 11, 2016 [Epub ahead of print].

12. Jaklitsch MT, Jacobson FL, Austin JH, Field JK, Jett JR, Keshavjee S, et al. The American Association for Thoracic Surgery guidelines for lung cancer screening using low-dose computed tomography scans for lung cancer survivors and other high-risk groups. J Thorac Cardiovasc Surg. 2012;144:33-8. 